\title{
Guarded and Banded Semigroups
}

\author{
Ernie Manes
}

\begin{abstract}
The variety of guarded semigroups consists of all $(S, \cdot,-)$ where $(S, \cdot)$ is a semigroup and $x \mapsto \bar{x}$ is a unary operation subject to four additional equations relating it to multiplication. The semigroup $P f n(X)$ of all partial transformations on $X$ is a guarded semigroup if $x \bar{f}=x$ when $x f$ is defined and is undefined otherwise. Every guarded semigroup is a subalgebra of $\operatorname{Pfn}(X)$ for some $X$. A covering theorem of McAlister type is obtained. Free guarded semigroups are constructed paralleling Scheiblich's construction of free inverse semigroups. The variety of banded semigroups has the same signature but different equations. There is a canonical forgetful functor from guarded semigroups to banded semigroups. A semigroup underlies a banded semigroup if and only if it is a split strong semilattice of right zero semigroups. Each banded semigroup $S$ contains a canonical subsemilattice $\boldsymbol{g}_{\star}(S)$. For any given semilattice $L$, a construction to synthesize the general banded semigroup $S$ with $\boldsymbol{g}_{\star}(S) \cong L$ is obtained.
\end{abstract}

Key words and phrases: Ample semigroups, inverse semigroups, restriction categories, varieties of semigroups with unary operation, ordered semigroups.

2005 Mathematics Subject Classification: primary: 20M07, secondary: 06F05.

\section{Introduction}

Inverse semigroups have been generalized by dropping regularity. This has led to left ample semigroups (introduced in [8] where they are called left type $A$ and subsequently studied in $[9,10,11,15]$ ), weakly left ample semigroups $([12,13,14])$ and, in this paper, guarded semigroups.

Each of these structures can be characterized by how they embed in a semigroup of partial transformations. Let $P f n(X)$ denote the monoid of partial transformations $X \rightarrow X$ with composition $x(f g)=(x f) g,(x \in X)$. Denote the submonoid of injective partial transformations by $\mathcal{J}_{X}$. For $f \in \operatorname{Pfn}(X)$, let $\operatorname{dom}(f)=\{x \in X: x f$ is defined $\}$. For $A \subset X$ the guard of $A$ is $\boldsymbol{g}_{A} \in \mathcal{J}_{X}$ with $\operatorname{dom}\left(\boldsymbol{g}_{A}\right)=A, x \boldsymbol{g}_{A}=x,(x \in A)$. A subset $S \subset P f n(X)$ is closed under guards if $f \in S \Rightarrow \boldsymbol{g}_{\operatorname{dom}(f)} \in S$ and $S$ is replete if $f \in S$ and $f^{2}=f \Rightarrow f$ is a guard. Note that $\mathcal{J}_{X}$ is replete.

The following results are well known.

Proposition 1.1 $S$ is an inverse semigroup if and only if, for some $X, S$ is isomorphic to a subsemigroup of $\mathcal{J}_{X}$ which is closed under inverses.

Proposition 1.2 $S$ is a left ample semigroup if and only if, for some $X, S$ is isomorphic to a subsemigroup of $\mathcal{J}_{X}$ which is closed under guards.

Proposition 1.3 $S$ is a weakly left ample semigroup if and only if, for some $X, S$ is isomorphic to a replete subsemigroup of $P f n(X)$ which is closed under guards. 
Notice that in $\mathcal{J}_{X}, \boldsymbol{g}_{\text {dom(f) }}=f f^{-1}$. It follows that inverse $\Rightarrow$ left ample $\Rightarrow$ weakly left ample.

While repleteness is unavoidable in $\mathcal{J}_{X}$, it is a restrictive condition for subsemigroups of $P f n(X)$, so it is natural to ask which semigroups are a guard-closed subsemigroup of $P f n(X)$ with no repleteness condition. At first glance, the question seems uninteresting since the endomorphism monoid of $X$ (of total transformations) is guard-closed (because each guard is the identity transformation) so that every monoid can be so embedded.

But there is another way to look at this. Each weakly left ample semigroup admists a canonical unary operation $x \mapsto x^{+}$which abstracts the guard operator $f \mapsto \boldsymbol{g}_{\operatorname{dom}(f)}$. Denote by $\mathcal{V}$ the variety of universal algebras $(S, \cdot,-)$ where $(S, \cdot)$ is a semigroup and $x \mapsto \bar{x}$ is an arbitrary unary operation. With $\bar{x}=x^{+}$, left ample and weakly left ample constitute quasivarieties of $\mathcal{V}([14$, Lemmas 2.2, 2.3]). The revised question is this: which algebras in $\mathcal{V}$ are isomorphic to a guard-closed subsemigroup of $\operatorname{Pfn}(X)$ for some $X$ ?

This question we answer successfully: guarded semigroups, a variety in $\mathcal{V}$ determined by four equations. The prototypical example is $\operatorname{Pfn}(X)$ with $\bar{f}=\boldsymbol{g}_{\operatorname{dom}(f)}$. Extremal examples are monoids where $\bar{x}$ is constant and semilattices where $\bar{x}=x$.

We show in Proposition 2.17 that a weakly left ample semigroup with $\bar{x}=x^{+}$is a guarded semigroup.

A $\mathcal{V}$-morphism $f$ is $\boldsymbol{g}$-idempotent-separating if $\bar{x} \neq \bar{y} \Rightarrow \bar{x} f \neq \bar{y}$. For weakly left ample semigroups, this is the same as idempotent-separating. The resulting theorem of McAlister type (Theorem 4.6 below) is as follows: Every guarded semigroup $S$ is a $\boldsymbol{g}$-idempotent-separating image of a guarded subsemigroup $H$ of a semidirect product of a monoid with a semilattice and such $H$ may be taken finite if $S$ is. Say that a guarded semigroup is $\boldsymbol{g}$-proper if given $\bar{x}=\bar{y}, e=\bar{e}$, ex $=e y$ then $x=y$. For left ample semigroups this coincides with proper. In the covering theorem just given, $H$ is $\boldsymbol{g}$-proper.

If $S$ is a guarded semigroup then $S^{1}$ with $\overline{1}=1$ is again a guarded semigroup, so it is fair to say that guarded semigroups with a unit constitute a broad class. The even broader class of guarded semigroups with a given left unit $u$ can be axiomatized as a variety of algebras $(S, \cdot, u, \star)$ with $(S, \cdot, u)$ a semigroup with left unit and $(S, \star)$ a right normal band subject to three further axioms. This leads to the concept of a banded semigroup which is part of the structure of any guarded semigroup.

Banded semigroups are semigroups with additional structure. In Theorem 8.10 below, we are able to characterize those semigroups which admit a banded structure as precisely the split strong semilattices of right zero semigroups.

Every guarded semigroup has a natural partial order. In Theorem 3.7 we show that, while a semigroup can be a guarded semigroup in more than one way, an ordered semigroup admits at most one guarded semigroup structure whose natural order coincides with the given order.

A banded semigroup $B$ also has a natural partial order and a canonical subset $\boldsymbol{g} \star(B)$ which is a meet semilattice under this order. Given an arbitrary meet semilattice $L$, we show in Theorem 9.1 how to construct the general solution $B$ of $\boldsymbol{g} \star(B) \cong L$.

On an arbitrary semigroup $S$, Green's $\mathcal{R}$-order induces a topology by virtue of which $S^{1}$ is a left topological monoid. As does any left topological monoid, such $S^{1}$ acts naturally on the union semilattice of the closures of finite sets. Any semidirect product of a semilattice with a monoid has a natural guard structure and the free guarded semigroup generated by $S$ is a guarded subsemigroup of the semidirect product resulting from the $S^{1}$-action just given. In particular, the free guarded semigroup generated by a set obtains by letting $S$ be the free semigroup generated by $X$.

Many questions remain open. A few are discussed at the end of the paper.

We thank the referee for valuable advice.

Added in proof: We have recently learned that guarded semigroups were considered (without 
proofs) in a paper by Batbedat in Springer Lecture Notes in Mathematics 855, 1981, and that Theorem 4.1 below was proved by Jackson and Stokes in this journal, 62, 2001. A forthcoming paper with Robin Cockett will provide details.

\section{Guarded Semigroups}

The notion of a guarded semigroup arises by adapting the axioms for a restriction category of Cockett and Lack [2]. Their axioms (R.1,..,R.4) are given below as $(\boldsymbol{g} .1, \ldots, \boldsymbol{g} .4)$, but note that we have reversed the order of composition to be consistent with the literature that we have cited. Their paper also develops the elementary consequences of the axioms. Other overlap between the two papers is explicitly cited below.

The most powerful of the axioms is $(\boldsymbol{g} .4)$. We believe the first occurrence in the semigroup literature is in [8] where it appears in the weaker form $a e=\overline{a e} a$ when $e$ is idempotent in a context where necessarily $e=\bar{e}$. In the category literature, the axiom first appears in [4].

Definition 2.1 A guard operator $x \mapsto \bar{x}$ on a semigroup $S$ satisfies the three equations

$(g .1) \quad \bar{x} x=x$

$(g .2) \quad \bar{x} \bar{y}=\bar{y} \bar{x}$

(g.3) $\overline{\bar{x} y}=\bar{x} \bar{y}$

The expected fourth axiom $(\boldsymbol{g} .4)$ will be given shortly. The numbering $(\boldsymbol{g} .5),(\boldsymbol{g} .6), \ldots$ will be used for basic immediate consequences of the axioms.

We have favored the Cockett and Lack notation $\bar{x}$ rather than the $x^{+}$which is used in the literature cited because of an anticipated interaction with the theory of lattice-ordered groups ([3]) where the notation $x^{+}=x \vee 1$ is well-established. This work will appear elsewhere.

Proposition 2.2 A guard operator satisfies the additional equations

$(g .5) \quad \bar{x}=\bar{x}$

(g.6) $\overline{x y} \bar{x}=\overline{x y}$

$(g .7) \quad \overline{\bar{x}}=\bar{x}$

(g.8) $\quad \overline{\bar{x}} \bar{y}=\bar{x} \bar{y}$

Proof $\quad(\boldsymbol{g} .5) \bar{x} \bar{x}=\overline{\bar{x} x}(\boldsymbol{g} .3)=\bar{x}(\boldsymbol{g} .1)$;

$(\boldsymbol{g} .6) \overline{x y} \bar{x}=\bar{x} \overline{x y}(\boldsymbol{g} .2)=\overline{\bar{x} x y}(\boldsymbol{g} .3)=\overline{x y}(\boldsymbol{g} .1)$

$(\boldsymbol{g} .7) \overline{\bar{x}}=\overline{\bar{x}} \bar{x}(\boldsymbol{g} .5)=\bar{x} \overline{\bar{x}}(\boldsymbol{g} .3)=\overline{\bar{x}} \bar{x}(\boldsymbol{g} .2)=\bar{x}(\boldsymbol{g} .1)$

$(\boldsymbol{g} .8) \overline{\bar{x}} \bar{y}=\bar{x} \overline{\bar{y}}(\boldsymbol{g} .3)=\bar{x} \bar{y}(\boldsymbol{g} .7)$

By the preceding,

$$
\boldsymbol{g}(S)=\{\bar{x}: x \in S\}=\{x \in S: x=\bar{x}\}
$$

is a semilattice with infimum $x y$. Elements of $\boldsymbol{g}(S)$ are guards.

Definition 2.3 For a guard operator on a semigroup, say that $x$ is deterministic if for all $y$

$$
x \bar{y}=\overline{x y} x
$$


Example 2.4 Let $\operatorname{Rel}(X)$ be the semigroup of all relations $R \subset X \times X$ with the usual composition

$$
R S=\{(x, z):(\exists y)(x, y) \in R,(y, z) \in S\}
$$

Let $x R$ denote $\{y:(x, y) \in R\}$ and define

$$
\bar{R}=\{(x, x): x R \neq \emptyset\}
$$

It is routine to see that $\operatorname{Rel}(X)$ is a semigroup with guard operator $\bar{R} . R$ is deterministic if and only if $R$ is a partial transformation, that is, $x R$ has at most one element for all $x$. To check this, first assume that $R$ is deterministic and that $(x, y),(x, z) \in R$. If $S=\{(y, y)\},(x, x) \in \overline{R S}$ so that $(x, z) \in \overline{R S} R=R \bar{S}$ which gives that $y=z$. Conversely, if $R$ is a partial transformation then, for all $S,(x, y) \in \overline{R S} R \Leftrightarrow x R S \neq \emptyset$ and $y=x R \Leftrightarrow(x, y) \in R \bar{S}$.

Definition 2.5 Let $S$ be a semigroup with guard operator. A subset $A \subset S$ is $\boldsymbol{g}$-full if $\boldsymbol{g}(S) \subset A$.

Proposition 2.6 Let $S$ be a semigroup with guard operator. Define

$$
\operatorname{det}(S)=\{x \in S: x \text { is deterministic }\}
$$

Then $\operatorname{det}(S)$ is a $\boldsymbol{g}$-full subsemigroup of $S$.

Proof For $x, y \in S, \bar{x} \bar{y}=\bar{x} \bar{y} \bar{x}$ (semilattice) $=\overline{\bar{x} y} \bar{x}(\boldsymbol{g} .3)$, and this shows $\boldsymbol{g}$-full. For $x, y, z \in S$,

$$
(x y) \bar{z}=x(y \bar{z})=x(\overline{y z} y)=\overline{x y z} x y
$$

shows $x y \in \operatorname{det}(S)$ if $x, y \in \operatorname{det}(S)$.

We are ready for the main definition of the paper.

Definition 2.7 A guarded semigroup, g-semigroup for short, is a semigroup with guard operator for which every element is deterministic. Thus $(\boldsymbol{g} .1, \ldots, \boldsymbol{g} .4)$ axiomatize $\boldsymbol{g}$-semigroups, where

$(g .4) x \bar{y}=\overline{x y} x$

If $S, T$ are $\boldsymbol{g}$-semigroups, a semigroup homomorphism $f: S \rightarrow T$ is a $\boldsymbol{g}$-homomorphism if also $\bar{x} f=\overline{x f}$. A g-subsemigroup $H$ of a $\boldsymbol{g}$-semigroup also satisfies $x \in H \Rightarrow \bar{x} \in H$.

$\boldsymbol{g}$-semigroups and $\boldsymbol{g}$-homomorphisms constitute an equationally-definable class with one binary operation and one unary operation. We hence denote this variety by $\mathcal{G S}$. A $\boldsymbol{g}$-monoid is a $\boldsymbol{g}$ semigroup with unit 1 . The corresponding $\boldsymbol{g}$-monoid homomorphisms also satisfy $1 f=1$, and we denote this variety as $\mathcal{G M}$.

Lemma 2.8 In any guarded semigroup, the following equation holds:

$(g .9) \overline{x \bar{y}}=\overline{x y}$

Proof $\quad$ By $(\boldsymbol{g} .4, \boldsymbol{g} .3, \boldsymbol{g} .6), \overline{x \bar{y}}=\overline{\overline{x y} x}=\overline{x y} \bar{x}=\overline{x y}$.

Example 2.9 It is evident from Proposition 2.6 that for any semigroup $S$ with guard operator, $\operatorname{det}(S)$ is a $\boldsymbol{g}$-semigroup. In the context of Example 2.4 this gives the $\boldsymbol{g}$-semigroup $\operatorname{Pfn}(X)$ with $\bar{f}=\boldsymbol{g}_{D o m(f)}$. 
We note that in a $\boldsymbol{g}$-monoid, $\overline{1}=\overline{1} 1=1$.

The forgetful functor from monoids to semigroups has a left adjoint which maps $S$ to $S^{1}$. This is obvious. The same construction produces the left adjoint of the forgetful functor from $\mathcal{G M}$ to $\mathcal{G S}$ : if $S$ is a guarded semigroup, $S^{1}$ is a guarded monoid defining $\overline{1}=1$. Thus one can work with either guarded semigroups or guarded monoids, depending on convenience.

Given a $\boldsymbol{g}$-semigroup in which $\bar{x}$ is constant, $(\exists u \forall x) \bar{x}=u, u$ is necessarily a monoid unit as follows. $u x=\bar{x} x=x, x u=x \bar{u}=\overline{x u} x=u x=x$.

Example 2.10 An arbitrary monoid is a $\boldsymbol{g}$-monoid under the definition $\bar{x}=1$, as is routinely checked.

We say a $\boldsymbol{g}$-semigroup is a monoid if $\bar{x}$ is constant.

Example 2.11 An arbitrary commutative band is a $\boldsymbol{g}$-semigroup if $\bar{x}=x$.

We say such a $\boldsymbol{g}$-semigroup is a semilattice. Clearly, a $\boldsymbol{g}$-semigroup is a semilattice if and only if $\boldsymbol{g}(S)=S$.

Example 2.12 Every inverse semigroup is a guarded semigroup with $\bar{x}=x x^{-1}$.

A $\boldsymbol{g}$-semigroup is an inverse semigroup if its underlying semigroup is and $\bar{x}=x x^{-1}$.

Proposition 2.13 (cf. [2, Pages 239-240]) Let $S$ be a g-semigroup. Then $S$ has a largest inverse subsemigroup, namely

$$
I(S)=\{x \in S:(\exists a \in S) x a x=x, x a, a x \in \boldsymbol{g}(S)\}
$$

and the set $E(I(S))$ of idempotents of $I(S)$ coincides with $g(S)$ so that $I(S)$ is $\boldsymbol{g}$-full in particular.

Proof Let $x a x=x, y b y=y, x a, a x, y b, b y \in \boldsymbol{g}(S)$ so that $x, y$ are typical elements of $I(S)$. Then

$$
x y(b a) x y=x(y b)(a x) y=x(a x)(y b) y=x y
$$

and

$$
x y b a=x \overline{y b} a=\overline{x y b} x a=\overline{x y b} \overline{x a} \in \boldsymbol{g}(S)
$$

Similarly, baxy $\in \boldsymbol{g}(S)$. This shows $I(S)$ is a subsemigroup. Let $u^{2}=u \in I(S)$ and choose $a$ with $u a u=u, u a, a u \in \boldsymbol{g}(S)$. Define $b=a u a$, so that $b, u$ are inverse. We have $u b=u a u a=u a \in \boldsymbol{g}(S)$ and $b u=a u a u=a u \in \boldsymbol{g}(S)$. Thus $b=a u a=(a u)(u a) \in \boldsymbol{g}(S)$. In particular, $b^{2}=b$ so $u=(u b)(b u) \in \boldsymbol{g}(S)$. Thus $I(S)$ as a semigroup is a regular semigroup in which idempotents (being guards) commute, and hence is an inverse semigroup. For $x \in S$,

$$
\bar{x}=\overline{x x^{-1} x}=\overline{\overline{x x^{-1}} x}=\overline{x x^{-1}} \bar{x}=\bar{x} \overline{x x^{-1}}=\overline{\bar{x} x x^{-1}}=\overline{x x^{-1}}=x x^{-1}
$$

so $I(S)$ as a $\boldsymbol{g}$-semigroup is an inverse subsemigroup. As every idempotent of $I(S)$ has form $x x^{-1}$, it follows $E(I(S))=\boldsymbol{g}(S)$. Finally, let $H$ be an inverse subsemigroup of $S$. For $x \in H, x x^{-1} x=x$ and $x x^{-1}=\bar{x} \in \boldsymbol{g}(S)$. Replacing $x$ with $x^{-1}, x^{-1} x \in \boldsymbol{g}(S)$. This shows $H \subset I(X)$.

From the definition of $I(S)$, it is clear that if the $\boldsymbol{g}$-semigroup $S$ is a monoid, $I(S)$ is the group of units of $S$. On the other hand, if $S$ is a semilattice then $I(S)=S$.

Proposition 2.14 Let $S$ be a $\boldsymbol{g}$-semigroup. Then the semigroup center $Z(S)$ is a $\boldsymbol{g}$-subsemigroup. 
Proof Let $x \in Z(S)$. Then for all $a \in S$,

$$
\begin{aligned}
a \bar{x} & =\overline{a x} a \quad(\boldsymbol{g} \cdot 4)=\overline{x a} a \quad(x \in Z(S)) \\
& =\overline{x \bar{a}} a \quad(\boldsymbol{g} \cdot 9) \\
& =\overline{\bar{a} x} a \quad(x \in Z(S))=\bar{a} \bar{x} a \quad(\boldsymbol{g} .3) \\
& =\bar{x} \bar{a} a \quad(\boldsymbol{g} .2)=\bar{x} a \quad(\boldsymbol{g} .1)
\end{aligned}
$$

so, having used all four axioms to show it, $\bar{x} \in Z(S)$ as well.

We conclude this section by characterizing weakly left ample semigroups as guarded semigroups. Similar results for inverse semigroups and left ample semigroups will be provided as Propositions $3.8,4.3$.

Various characterizations of weakly left ample semigroups exist. We take [12, Page 723] as our definition.

Definition 2.15 A semigroup equipped with a unary operation $\bar{x}$ is a weakly left ample semigroup if the following equations and equational implications hold.

(wla.1) $\bar{x} \bar{x}=\bar{x}$

(wla.2) $\bar{x} x=x$

(wla.3) idempotents commute

(wla.4) $x^{2}=x, x y=y \Rightarrow x \bar{y}=\bar{y}$

(wla.5) $\bar{x}=\bar{y} \Rightarrow \overline{z x}=\overline{z y}$

(wla.6) $x^{2}=x \Rightarrow y x=\overline{y x} y$

Although not obvious from the above definition, a semigroup can be weakly left ample in at most one way. If $a \widetilde{\mathcal{R}} b$ means $(\forall e \in E(S)) e a=a \Leftrightarrow e b=b$ then $\widetilde{\mathcal{R}}$ is an equivalence relation and $\bar{x}$ is the unique idempotent in the $\widetilde{\mathcal{R}}$-class of $x$. Note, however, that we prove the next proposition using only (wla.1,...,wla.6).

Definition 2.16 A $\boldsymbol{g}$-semigroup is replete if $E(S)=\boldsymbol{g}(S)$, that is, if every idempotent is a guard.

Proposition 2.17 Let $S$ be a semigroup with unary operation $\bar{x}$. Then $S$ is weakly left ample if and only if $S$ is a replete $\boldsymbol{g}$-semigroup.

Proof First assume (wla.1,...,wla.6) hold. Let $e \in E(S)$. As $e^{2}=e, e \bar{e}=\bar{e}$, so $\bar{e}=e \bar{e}=\bar{e} e=e$. In particular, $\overline{\bar{x}}=\bar{x}$. We can now check the $\boldsymbol{g}$-semigroup axioms. (g.1) is (wla.2), (g.2) follows from (wla.1, wla.3) and (g.4) is immediate from (wla.1, wla6). For $(\boldsymbol{g} .3), \bar{x}=\overline{\bar{x}} \Rightarrow \overline{x \bar{y}}=\overline{\bar{x}} \bar{y}$ (by (wla.5)) and this is $\bar{x} \bar{y}$ because (wla.3) implies that the product of idempotents is idempotent. Conversely, let $S$ be a $\boldsymbol{g}$-semigroup with $E(S)=\boldsymbol{g}(S)$. (wla.1, wla.2, wla.3, wla.6) are clear. For (wla.4), if $x y=y$ with $x=\bar{x}, x \bar{y}=\bar{x} \bar{y}=\overline{\bar{x} y}=\overline{x y}=\bar{y}$. For (wla.5), if $\bar{x}=\bar{y}$ then $\overline{z x}=\overline{z \bar{x}}=\overline{z \bar{y}}=\overline{z y}$.

\section{The Natural Order}

A semigroup can be a $\boldsymbol{g}$-semigroup in more than one way. For example, $P f n(X) \operatorname{admits} \bar{f}=\boldsymbol{g}_{\operatorname{Dom}(f)}$ and $\bar{f}=i d_{X}$ as distinct guarded semigroup structures. We show in this section, however, that $\boldsymbol{g}$-semigroups are a class of ordered semigroups. 
Definition 3.1 Let $S$ be a g-semigroup. The natural order on $S$ is

$$
x \leq y \Leftrightarrow \bar{x} y=x
$$

Restricted to $I(S)$, the natural order is the usual one for inverse semigroups. Restricted to $\boldsymbol{g}(S)$ it is also the usual order on idempotents.

Proposition 3.2 ([2, Section 2.1.4]) The natural order is a compatible partial order and $\boldsymbol{g}$-homomorphisms are monotone.

Proof $x \leq x$ by $(\boldsymbol{g} .1)$. If $x \leq y \leq x$ then

$$
x=\bar{x} y=\bar{x} \bar{y} x=\bar{y} \bar{x} x=\bar{y} x=y
$$

which gives antisymmetry. If $x \leq y \leq z$ then

$$
\bar{x} z=\overline{\bar{x} y} z=\bar{x} \bar{y} z=\bar{x} y=x
$$

so $x \leq z$. Fix $a$ and let $x \leq y$. Then

$$
\overline{x a} y a=\overline{x a} \bar{x} y a(\boldsymbol{g} \cdot 6)=\overline{x a} x a=x a
$$

shows $x a \leq y a$. Further,

$$
\overline{a x} a y=a \bar{x} y=a y
$$

so $a x \leq a y$. This completes the proof that $\leq$ is a compatible partial order. If $f: S \rightarrow T$ is a $\boldsymbol{g}$-homomorphism and $x \leq y$ in $S$ then $\overline{x f} y f=(\bar{x} y) f=x f$ so $x f \leq y f$ and $f$ is monotone.

Mitsch [24] defines a partial order $\leq_{M}$ on an arbitrary semigroup by

$$
x \leq_{M} y \Leftrightarrow\left(\exists t, u \in S^{1}\right) x=t y=y u, t x=x
$$

If $x \leq y$, set $t=\bar{x}$ to get $x=t y=t x$. This begs for a comparison of the two orders. For a monoid as in Example 2.10, $x \leq y \Leftrightarrow x=y$ whereas $x \leq_{M} y$ is often nontrivial. In $P f n(X)$, if $g$ is a constant total transformation and if $\emptyset \neq f<g$, no $h$ exists with $f=g h$ so $f \leq_{M} g$. In general, $\leq_{M}$ is not a compatible partial order. Note, also, that $\leq_{M}$ is determined by the semigroup structure alone whereas $\leq$ is not. So, one concludes in the main, that $\leq$ and $\leq_{M}$ behave quite differently.

Observe that (g.6) asserts that $\overline{x y} \leq \bar{x}$.

Generalizing the terminology of [23], we have

Proposition 3.3 The natural order on a $\boldsymbol{g}$-semigroup is amenable, that is, $x \leq y \Rightarrow \bar{x} \leq \bar{y}$.

Proof $\overline{\bar{x}} \bar{y}=\bar{x} \bar{y}=\overline{\bar{x} y}=\bar{x}$.

Proposition 3.4 Let $S$ be a semigroup, $e \in \boldsymbol{g}(S), A=\{x: \bar{x}=e\}$. Then $A$ is discretely ordered under the natural order.

Proof Let $x, y \in A, x \leq y$. Then $x=\bar{x} y=\bar{y} y=y$.

Definition 3.5 Let $(X, \leq)$ be a poset, $A \subset X$. A is a lower set if $x \leq a$ with $a \in A$ implies $x \in A$. Dually, $A$ is an upper set if $a \leq x$ with $a \in A$ implies $x \in A$.

Proposition 3.6 Let $S$ be a $\boldsymbol{g}$-semigroup, $H$ a $\boldsymbol{g}$-full $\boldsymbol{g}$-subsemigroup of $S$. Then $H$ is a lower set. 
Proof Let $x \leq h$ with $h \in H$. Then as $x=\bar{x} h$ and $\bar{x} \in H, x \in H$.

Recall that the negative cone of a partially ordered semigroup is $\{x: \forall y x y \leq y, y x \leq y\}$.

Theorem 3.7 Let $S$ be a $\boldsymbol{g}$-semigroup. Then $\boldsymbol{g}(S)$ is the negative cone of $S$. Moreover,

$$
\bar{x}=\operatorname{Min}\{e \in \boldsymbol{g}(S): e x=x\}
$$

so the guard operator is determined by its natural order.

Proof Because $\bar{x} y=\bar{x} \bar{y} y=\bar{x} y, \bar{x} y \leq y$. Also, $y \bar{x} \leq y$ because $\bar{y} \bar{x} y=\overline{y x} y(\boldsymbol{g} \cdot 9)=y \bar{x}$. Conversely, if $x$ is in the negative cone then $x=\bar{x} x \leq \bar{x}$ so, by Proposition 3.4, $x=\bar{x}$. This shows that the negative cone is precisely $\boldsymbol{g}(S)$. If $e \in \boldsymbol{g}(S)$ with $e x=x$ then $e \bar{x}=\bar{e} \bar{x}=\overline{\bar{e} x}=\overline{e x}=\bar{x}$, so $\bar{x} \leq e$.

The preceding theorem establishes $\mathcal{G S}$ as a subcategory of the category of partially ordered semigroups and monotone homomorphisms, although it is not a full subcategory.

Notice that in any guarded semigroup, $\boldsymbol{g}(S)$ is a lower set. For this is true of the negative cone in any partially ordered semigroup.

We next apply the natural order to characterize inverse semigroups within guarded semigroups.

Proposition 3.8 Let $S$ be a $\boldsymbol{g}$-semigroup. Then $S$ is an inverse semigroup if and only if $S$ is regular and replete.

Proof That an inverse semigroup is regular is clear and, since all idempotents have form $x x^{-1}=\bar{x}$ for some $x$, it is replete as well. Conversely, if $S$ is regular and $E(S)=\boldsymbol{g}(S)$, idempotents commute so that the underlying semigroup of $S$ is inverse, but we must also prove that $\bar{x}=x x^{-1}$. To that end, as $\bar{x}=\bigwedge\left\{e: e^{2}=e, e x=x\right\}$, as $x x^{-1} x=x$, and as $x x^{-1}$ is idempotent, $\bar{x} \leq x x^{-1}$. Further,

$$
\overline{x x^{-1}} \bar{x}=x x^{-1} \bar{x}^{-1}=x(\bar{x} x)^{-1}=x x^{-1}
$$

gives $x x^{-1} \leq \bar{x}$.

\section{Fundamental Structure Theorems}

The following Wagner/Preston theorem (at least for $\boldsymbol{g}$-monoids) appears in [2, Theorem 3.9] as a corollary of a much more extensive theorem for categories. We offer here a straightforward proof in the style of the usual theorem for inverse semigroups.

Theorem 4.1 Every $\boldsymbol{g}$-semigroup $S$ is isomorphic to a $\boldsymbol{g}$-subsemigroup of $\operatorname{P} \operatorname{fn}(S)$.

Proof Define $\rho: S \rightarrow \operatorname{Pfn}(S), a \mapsto \rho_{a}$ by

$$
x \rho_{a}=\left\{\begin{array}{cc}
x a & \text { if } x \in D_{a} \\
\perp & \text { otherwise }
\end{array}\right.
$$

where $\perp$ means "undefined" and $D_{a}=\operatorname{Dom}\left(\rho_{a}\right)$ is yet to be determined. As $\rho$ is to preserve guards, $\rho_{\bar{a}}$ must be the same as $\overline{\rho_{a}}$. Thus $x \rho_{\bar{a}}$ is $x \bar{a}$ for $x \in D_{\bar{a}}$ and $x \overline{\rho_{a}}$ is $x$ for $x \in D_{a}$. This strongly suggests that we define

$$
D_{a}=\{x: x \bar{a}=x\}
$$


and we do so. Then $D_{\bar{a}}=\{x: x \overline{\bar{a}}=x\}=D_{a}$ and so $\rho$ preserves guards. Since $x(a b)=(x a) b$, $\rho_{a b}=\rho_{a} \rho_{b}$ providing both transformations have the same domain. Now

$$
\begin{aligned}
x \rho_{a b} \text { is defined } & \Leftrightarrow x \overline{a b}=x \\
x \rho_{a} \rho_{b} \text { is defined } & \Leftrightarrow x \bar{a}=x \text { and } x a \bar{b}=x a
\end{aligned}
$$

First suppose that $x \rho_{a b}$ is defined. Then $x \bar{a}=x \overline{a b} \bar{a}=x \overline{a b}=x$ and $x a \bar{b}=x \overline{a b} a=x a$, so $x \rho_{a} \rho_{b}$ is defined. Conversely, suppose that $x \rho_{a} \rho_{b}$ is defined. Then

$$
x \overline{a b}=\overline{x a b} x=\overline{x a \bar{b}} x=\overline{x a} x=\overline{x \bar{a}} x=\bar{x} x=x
$$

Thus $\rho$ is a $\boldsymbol{g}$-homomorphism and it remains only to show $\rho$ is injective. Let $\rho_{a}=\rho_{b}$ so that $D_{a}=D_{b}$ and $x a=x b$ for $x \in D_{a}$. As $\bar{a} \bar{a}=\bar{a}, \bar{a} \in D_{a}$. Thus $a=\bar{a} a=\bar{a} b$ which shows $a \leq b$. Symmetrically, $b \leq a$.

For inverse semigroups, the proof of the above theorem is similar, using $D_{a}=S a^{-1}$. Noting that $\bar{a}=a a^{-1}$, it is routine to check that $S a^{-1}=\{x: x \bar{a}=x\}$. Thus for a guarded semigroup that happens to be an inverse semigroup, the usual proof of the Wagner/Preston theorem is obtained from the proof above, although one has to further check closure under inverses.

Definition 4.2 A left ample semigroup is a $\boldsymbol{g}$-semigroup isomorphic to a $\boldsymbol{g}$-subsemigroup of the $\boldsymbol{g}$-semigroup $\mathcal{J}_{X}$ of injective partial transformations.

Proposition 4.3 A g-semigroup is left ample if and only if it satisfies the equational implication

$$
x \bar{a}=x, y \bar{a}=y, x a=y a \Rightarrow x=y
$$

Proof Suppose $f, g, h \in \mathcal{J}_{X}$ with $f \bar{h}=f, g \bar{h}=g, f h=g h$. Let $x f$ be defined. As $f \bar{h}=f$, $x f h$ is defined so that $x f h=x g h$. As $h$ is injective, $x f=x g$. This shows $f \leq g$. Symmetrically, $g \leq f$, so $f=g$. Thus the equational implication holds for $\mathcal{J}_{X}$, hence for any left ample semigroup. Conversely, consider the embedding $\rho$ of Theorem 4.1. Let $x \rho_{a}, y \rho_{a}$ be defined and equal so that $x \bar{a}=a, y \bar{a}=y, x a=y a$. By hypothesis, $x=y$. Thus $\rho$ takes values in $\mathcal{J}_{X}$.

A similar description of left ample semigroups as a quasivariety appears in [14, Lemma 2.2].

Semidirect products of appropriate monoids with semilattices have played a role on several fronts: free inverse semigroups [25], proper inverse semigroups [21, 22] and other proper semigroups ([7, 9] to cite but two). The same will be true for $\boldsymbol{g}$-semigroups. We begin with the basic construction.

Let $L$ be a commutative band so that $L$ is an inf semilattice with respect to $x \leq y \Leftrightarrow x y=x$ and a sup semilattice with respect to $x \leq y \Leftrightarrow x y=y$. Both interpretations will arise naturally later. Let $S$ be a monoid acting on $L$ on the left via

$$
\tau: S \times L \rightarrow L, \quad(s, e) \mapsto s \cdot e
$$

that is, the following equations hold.

$($ act.1) $(s t) \cdot e=s \cdot(t \cdot e)$

(act.2) $1 \cdot e=e$

$(\mathbf{a c t} .3) s \cdot(e f)=(s \cdot e)(s \cdot f)$

The resulting semidirect product of $L$ with $S$ is the semigroup structure on $L \times S$ given by

$$
(e, s)(f, t)=(e(s \cdot f), s t)
$$

It is well known that this is a semigroup, and we denote it as $L \bigotimes_{\tau} S$, dropping the subscript when it is clear from context or irrelevant. 
Proposition 4.4 A semidirect product $L \bigotimes S$ is a $\boldsymbol{g}$-semigroup if $\overline{(e, s)}=(e, 1)$.

Proof $(\boldsymbol{g} \cdot 1) \overline{(e, s)}(e, s)=(e, 1)(e, s)=(e(1 \cdot e), 1 s)=(e, s)$.

$(\boldsymbol{g} .2)(e, 1)(f, 1)=(e f, 1)=(f e, 1)=(f, 1)(e, 1)$.

$(\boldsymbol{g} .3) \overline{\overline{(e, s)}(f, t)}=\overline{(e, 1)(f, t)}=(e f, 1)=\overline{(e, s)} \overline{(e, t)}$.

$(\boldsymbol{g} \cdot 4)(e, s) \overline{(f, t)}=(e, s)(f, 1)=(e(s \cdot f), s)=(e(s \cdot f)(1 \cdot e), s)=(e(s \cdot f), 1)(e, x)=\overline{(e, s)(f, t)}(e, s)$.

Definition 4.5 A g-homomorphism $\psi: S \rightarrow T$ is $\boldsymbol{g}$-idempotent separating if $e \neq f \in \boldsymbol{g}(S) \Rightarrow$ $e \psi \neq f \psi$. If $\psi$ even induces an isomorphism $\boldsymbol{g}(S) \cong \boldsymbol{g}(T)$ then $\psi$ is strong $\boldsymbol{g}$-idempotent separating.

The following is a covering theorem of McAlister type for guarded semigroups. It asserts that arbitrary $\boldsymbol{g}$-semigroups can be built from the extremal cases, monoids (only one guard) and semilattices (every element is a guard).

Theorem 4.6 Every $\boldsymbol{g}$-semigroup $S$ is the image under a strong $\boldsymbol{g}$-idempotent separating $\boldsymbol{g}$-homomorphism of a full $\boldsymbol{g}$-subsemigroup $H$ of a semidirect product of a monoid with a semilattice. Moreover, $H$ can be taken finite if $S$ is.

Proof If $S$ is a $\boldsymbol{g}$-semigroup, $S^{1}$ acts on the semilattice $\boldsymbol{g}(S)$ by $(s, e) \tau=s \cdot e=\overline{s e}$. We check the action equations (act.1, act.2, act.3). $(s t) \cdot e=\overline{s t e}=\overline{s t \bar{e}}=s \cdot(t \cdot e) ; 1 \cdot e=\overline{1 e}=e ; s \cdot(e f)=\overline{s e f}$ whereas $(s \cdot e)(s \cdot f)=\overline{s e} \overline{s f}$. The two expressions are equal in $P f n(X)$ because if $e=\boldsymbol{g}_{B}, f=\boldsymbol{g}_{C}$, and $A=\{x: x s$ is defined, $x s \in B \cap C\}$, both sides are $\boldsymbol{g}_{A}$. But if the equation holds in all $\operatorname{Pfn}(X)$ it must hold in all $\boldsymbol{g}$-semigroups. Define

$$
H=\left\{(e, x) \in \boldsymbol{g}(S) \bigotimes_{\tau} S^{1}: e \leq \bar{s}\right\}
$$

(more precisely, if $s \in S, \bar{s}$ is the original guard whereas if $s=1, \overline{1}=1$. Hence we use $S^{1}$ as a monoid, ignoring the guard structure, to construct the semidirect product but use the guard structure to define the subset $H)$. If $e \leq \bar{s}, f \leq \bar{t}$ then $(e, s)(f, t)=(\overline{e s f}, s t)$ and, using the amenable property and $(\boldsymbol{g} .9)$,

$$
\overline{e s f} \leq \bar{s} \overline{s \bar{t}}=\bar{s} \overline{s t} \leq \overline{s t}
$$

so $H$ is a subsemigroup. For $e \in \boldsymbol{g}(S), e \leq \overline{1}=1$ since $e 1=e$, so $(e, 1) \in H$ and $H$ is a full $\boldsymbol{g}$-subsemigroup, finite if $S$ is. Define $\gamma: H \rightarrow S$ by

$$
(e, s) \gamma=e s
$$

Then

$$
((e, s)(f, t)) \gamma=(e \overline{s f}, s t) \gamma=e \overline{s f} s t=e s \bar{f} t=e s f t=(e, s) \gamma(f, t) \gamma
$$

Also, as $e \leq \bar{s}$,

$$
\overline{(e, s) \gamma}=\overline{e s}=\overline{e \bar{s}}=\bar{e}=e=(e, 1) \gamma=\overline{(e, s)} \gamma
$$

This shows so far that $\gamma$ is a $\boldsymbol{g}$-homomorphism. Given $s,(\bar{s}, s) \gamma=s$, so $\gamma$ is surjective. As $(1, e)(1, f)=(1, e f), \gamma$ induces an isomorphism $\boldsymbol{g}(H) \cong \boldsymbol{g}(S)$.

Corollary 4.7 The class of semidirect products $L \bigotimes_{\tau} S$, for $\tau$ a left action of the monoid $S$ on the semilattice $L$, satisfies no equations in the operations $x y, \bar{x}$ which are not satisfied by all $\boldsymbol{g}$ semigroups. 
It is well known that groups constitute a full reflective subcategory of inverse semigroups where the group reflection of a semigroup $S$ is $S / \sigma$ for the congruence $\sigma$ is given by

$$
x \sigma y \Leftrightarrow(\exists e) e^{2}=e, e x=e y
$$

It is obvious that all idempotents are in a single $\sigma$-class, so that $S / \sigma$ is an inverse semigroup with one idempotent, hence a group. The construction works as well for guarded semigroups, save that a guarded semigroup with one idempotent is a monoid. The formal statement is as follows, where a $\boldsymbol{g}$-congruence on a $\boldsymbol{g}$-semigroup $S$ is a $\boldsymbol{g}$-subsemigroup of $S \times S$ which is also an equivalence relation.

Proposition 4.8 Let $S$ be a guarded semigroup. Write $x \sigma y$ if there exists $e \in g(S)$ with ex $=e y$. Then $\sigma$ is a $\boldsymbol{g}$-congruence and $S / \sigma$ is the reflection of $S$ in the full subcategory of monoids in $\mathcal{G S}$.

Proof $\bar{x} x=\bar{x} x$ shows $\sigma$ is reflexive, and symmetric is obvious. If $e, f \in \boldsymbol{g}(S)$ and $e x=e y$, $f y=f z, e f x=f e x=f e y=e f y=e f z$, so $\sigma$ is an equivalence relation. If $x \sigma y$ choose $e \in \boldsymbol{g}(S)$ with $e x=e y$. Let $z \in S$. Then $e x z=e y z$. Also, $\overline{z e} z x=z \bar{e} x=z e x=z e y=\overline{z e} y$. Thus $\sigma$ is a semigroup congruence. Since $\boldsymbol{g}(S)$ is a subsemilattice, all guards are in one $\sigma$-class so $\sigma$ is a $\boldsymbol{g}$-congruence and $S / \sigma$ is a monoid. Now consider a $\boldsymbol{g}$-homomorphism $f: S \rightarrow M$ where $M$ is a monoid. We must show there exists a unique $\boldsymbol{g}$-homomorphism $\psi$ in the triangle

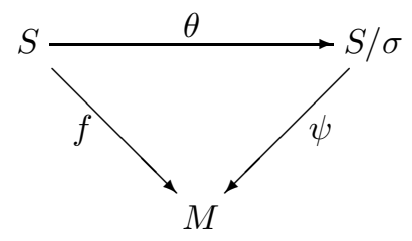

It is necessary and sufficient to show that if $x \sigma y$ then $x f=y f$. To that end, if $e \in \boldsymbol{g}(S)$ and $e x=e y$ then as $e f=\bar{x} f=\overline{e f}$ is the unit of $M$,

$$
x f=x f e f=(x e) f=(y e) f=y f
$$

Example 4.9 Let $L$ be a semilattice and let $S$ be a monoid. For any semidirect product $L \bowtie S$, $(e, s) \sigma(f, t) \Leftrightarrow s=t$. Thus the monoid reflection is the projection map $L \bowtie S \rightarrow S$.

\section{$5 \quad$ Proper Guarded Semigroups}

Lemma 5.1 Let $S$ be a $\boldsymbol{g}$-semigroup, $x, y \in S$. Then

$$
((\forall e \in \boldsymbol{g}(S)) e x=x \Leftrightarrow e y=y) \Leftrightarrow \bar{x}=\bar{y}
$$

Proof The left side implies the right because $\bar{x}=\bigwedge\{e \in \boldsymbol{g}(S): e x=x\}$. Conversely, if $\bar{x}=\bar{y}$ and $e x=x$ then $\bar{x} \leq e$ so $\bar{y} \leq e$ and $e y \leq y=\bar{y} y \leq e y$ shows $e y=y$.

Definition 5.2 A guarded semigroup is $\boldsymbol{g}$-proper if whenever $\bar{x}=\bar{y}$ and $(\exists e \in \boldsymbol{g}(S))$ ex $=e y$, then necessarily $x=y$. $S$ is $\boldsymbol{g}$-unitary if $\boldsymbol{g}(S)$ is an upper set.

$\boldsymbol{g}$-proper and $\boldsymbol{g}$-unitary coincide with proper and $E$-unitary for weakly left ample semigroups, left ample semigroups and inverse semigroups since these are replete. It is known [7, Example 3] that $\boldsymbol{g}$-unitary does not imply $\boldsymbol{g}$-proper. 
Proposition 5.3 A $\boldsymbol{g}$-proper guarded semigroup is $\boldsymbol{g}$-unitary.

Proof Let $e \in \boldsymbol{g}(S), e \leq s$. Then $\overline{\bar{s}}=\bar{s}$ and $e s=\bar{e} s=e$. As $e \leq s, e=\bar{e} \leq \bar{s}$ so, similarly, $e \bar{s}=e$. Thus $e s=e \bar{s}$. By $\boldsymbol{g}$-proper, $s=\bar{s}$.

Extremal guarded semigroups are $\boldsymbol{g}$-proper. If $S$ is a monoid, $\boldsymbol{g}(S)=\{1\}$ so if $e x=e y$ for some guard $e, x=y$. If $S$ is a semilattice, $\bar{x}=\bar{y} \Leftrightarrow x=y$ because $\bar{x}=x$. More generally we have

Example 5.4 $L \bigotimes S$ ( $L$ a semilattice, $S$ a monoid) is $\boldsymbol{g}$-proper. For $\overline{(e, s)}=\overline{(f, t)} \Leftrightarrow e=f$ and $\exists(g, 1)$ with $(g, 1)(e, s)=(g, 1)(f, t) \Rightarrow s=t$.

It is clear from the definition that $\boldsymbol{g}$-proper guarded semigroups form a quasivariety, so any $\boldsymbol{g}$ subsemigroup of a $\boldsymbol{g}$-proper semigroup is $\boldsymbol{g}$-proper. In particular, Theorem 4.6 shows that every guarded semigroup is a $\boldsymbol{g}$-idempotent separating image of a $\boldsymbol{g}$-proper guarded semigroup.

Example 5.5 Let $G$ be a lattice-ordered group. Let $S$ be a submonoid of $G$ and let $H$ be a convex subgroup of $G$. By [3, Theorem 8.2], the left coset space $G / H$ is an inf-semilattice with $x H \wedge y H=$ $(x \wedge y) H$. S acts on $G / H$ by $g \cdot(x H)=(g x) H$, as is well known. The resulting semidirect product $S \bowtie(G / H)$ is then a proper $\boldsymbol{g}$-semigroup. It is, in fact, left ample because $S$ is right cancellative, as is easy to check.

\section{$6 \quad$ Free Guarded Semigroups}

Cockett and Lack [2] constructed the free restriction category generated by a category, influenced by Scheiblich's construction of the free inverse semigroup in [25]. We use the same proof to construct the free $\boldsymbol{g}$-semigroup generated by a semigroup, but present it to show the use of some topological ideas and to highlight a connection with Green's $\mathcal{R}$ order. Of course, the free $\boldsymbol{g}$-semigroup generated by a set $X$ is just the free $\boldsymbol{g}$-semigroup generated by the free semigroup generated by $X$.

If $A$ is a subset of a topological space, we denote the closure of $A$ by $A^{\bullet}$. It is well known that a function $f$ between topological spaces is continuous if and only if $A^{\bullet} f \subset(A f)^{\bullet}$. We pause to establish two other versions of this which we need.

Lemma 6.1 Let $f: X \rightarrow Y$ be a function between topological spaces. Then the following conditions are equivalent to the continuity of $f$.

(cont.1) $A^{\bullet} f \subset(A f)^{\bullet}$

(cont.2) $A^{\bullet}=B^{\bullet} \Rightarrow(A f)^{\bullet}=(B f)^{\bullet}$

(cont.3) $\left(A^{\bullet} f\right)^{\bullet}=(A f)^{\bullet}$

Proof $\left(\right.$ cont.1) $\Rightarrow\left(\right.$ cont.2): If $A^{\bullet}=B^{\bullet}$ then $(A f)^{\bullet} \supset\left(A^{\bullet}\right) f=\left(B^{\bullet}\right) f \supset B f$ so that $(B f)^{\bullet} \subset(A f)^{\bullet}$, and the reverse inequality symmetrically. (cont.2) $\Rightarrow\left(\right.$ cont.3): $A^{\bullet}=A^{\bullet \bullet} \Rightarrow(A f)^{\bullet}=\left(A^{\bullet} f\right)^{\bullet}$. $\left(\right.$ cont.3) $\Rightarrow\left(\right.$ cont.1): $A^{\bullet} f \subset\left(A^{\bullet} f\right)^{\bullet}=(A f)^{\bullet}$.

Example 6.2 Let $S$ be a left topological monoid with unit 1, that is, a monoid equipped with a topology (no separation axioms assumed!) for which all left multiplications $\lambda_{a}, x \lambda_{a}=a x$, are continuous. Let $\mathcal{C}$ be the sup semilattice of closed subsets of $X$, the join being union. Then $S$ acts on $\mathcal{C}$ on the left by

$$
s \cdot A=(s A)^{\bullet}, \text { where } s A=\{s a: a \in A\}
$$


We check the action axioms. Since $\lambda_{a}$ is continuous it follows from (cont.3) that $\left(s(t A)^{\bullet}\right)^{\bullet}=(s t A)^{\bullet}$, and this is precisely (act.1). Since elements of $\mathcal{C}$ are closed, (act.2) is obvious, and (act.3) amounts to the Kuratowski axiom $(A \cup B)^{\bullet}=A^{\bullet} \cup B^{\bullet}$.

Definition 6.3 Let $\mathcal{P O}$ be the category of preordered sets $(X, \leq)$ (i.e., $\leq$ is reflexive and transitive) and monotone maps $(x \leq y \Rightarrow f x \leq f y)$. Let $\mathcal{T O P}$ be the category of topological spaces and continuous maps. It is known ([19]) that $\mathcal{P} \mathcal{O}$ is the full subcategory of $\mathcal{T} \mathcal{O} \mathcal{P}$ of all spaces with the property that every intersection of open sets is open -we call such spaces pospaces.

We review the constructions. Given preordered $(X, \leq)$, the pospace topology of $(X, \leq)$ has as its open sets precisely all the lower sets. Conversely, if $X$ is a pospace the corresponding preorder, called the specialization preorder, is defined by

$$
x \leq y \Leftrightarrow y \in\{x\}^{\bullet} \Leftrightarrow x \in U_{y}
$$

where $U_{y}$ is the intersection of all open neighborhoods of $y$. As is easily checked, the closed subsets are just the upper sets. More generally, $A^{\bullet}=\{x:(\exists a \in A) x \geq a\}$. Note that $\leq$ is antisymmetric if and only if the corresponding pospace is $T 0$. While many nontrivial results appear in [19], the facts stated here are easily established by the reader.

Green's $\mathcal{R}$ order is reflexive and transitive and so induces a pospace as follows.

Proposition 6.4 Let $S$ be an arbitrary semigroup. Then

$$
A^{\bullet}=\left\{y:\left(\exists x \in S^{1}\right), y x \in A\right\}
$$

is a Kuratowski closure operator on $S$. With this topology, $S$ is a left topological semigroup.

Proof The Green order $y \leq_{\mathcal{R}} z$ if $\left(\exists x \in S^{1}\right) y x=z$ is reflexive and transitive and satisfies $\forall w, y \leq_{\mathcal{R}} z \Rightarrow w y \leq_{\mathcal{R}} w z$. The given closure formula above is just the closure operator of the pospace topology. The left multiplications are monotone, as already noted, and so are continuous.

We apply the previous proposition to the construction of free guarded semigroups.

Theorem 6.5 ([2, Pages 232-235] Let $S$ be a semigroup. Regard $S^{1}$ as a left topological monoid via the green $\mathcal{R}$-order as in Proposition 6.4. Let $\mathcal{C} \otimes S^{1}$ be the semidirect product $\boldsymbol{g}$-semigroup resulting from Proposition 4.4 using the action of Example 6.2. Define

$$
H S=\left\{(A, x) \in \mathcal{C} \bigotimes S^{1}: x \in A \cup\{1\}, A=F^{\bullet} \text { for some finite } F\right\}
$$

Then $H S$ is a $\boldsymbol{g}$-subsemigroup and, via the inclusion of the generators $\eta: S \rightarrow H S, x \mapsto\left(\{x\}^{\bullet}, x\right)$, $H S$ is the free guarded semigroup generated by the semigroup $S$.

Proof $(A, x) \in H S \Rightarrow(A, 1) \in H S$ so $H S$ is closed under guards. We must check that $H S$ is a subsemigroup. Composition is given by

$$
\left.\left(F^{\bullet}, x\right)\left(G^{\bullet}, y\right)=\left(F^{\bullet} \cup\left(x G^{\bullet}\right)^{\bullet}\right), x y\right)=\left(F^{\bullet} \cup(x G)^{\bullet}, x y\right)(\text { cont.3 })=\left((F \cup x G)^{\bullet}, x y\right)
$$

and $F \cup x G$ is finite. If $x y \neq 1$ we must prove $x y \in F^{\bullet} \cup(x G)^{\bullet}$. If $x \neq 1=y, x y=x \in F^{\bullet}$. Otherwise, $y \in G^{\bullet}$ so $x y \in x G^{\bullet}$. So far, we have shown that $H S$ is a guarded semigroup. We next show that $\eta$ is a semigroup homomorphism. For convenience we start writing $a^{\bullet}$ instead of the more cumbersome $\{a\}^{\bullet}$. Since $x \in(x y)^{\bullet}$,

$$
(x \eta)(y \eta)=\left(x^{\bullet} \cup(x y)^{\bullet}, x y\right)=\left((x y)^{\bullet}, x y\right)=(x y) \eta
$$

To complete the proof, we must establish the universal property 


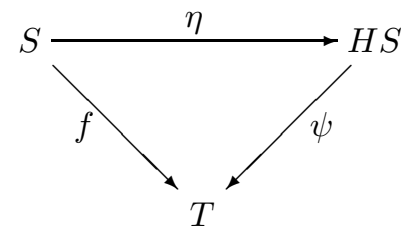

that for each guarded semigroup $T$ and semigroup homomorphism $f: S \rightarrow T$ there exists a unique $\boldsymbol{g}$-homomoprhism $\psi: H S \rightarrow T$ with $\eta \psi=f$. To this end, let $F=\left\{x_{1}, \ldots, x_{n}\right\} \subset S$ so that a typical element of $H S$ has form $\left(F^{\bullet}, x\right)$. If $x \neq 1$,

$$
\begin{aligned}
\left(F^{\bullet}, x\right) & =\left(\left\{x, x_{1}, \ldots, x_{n}\right\} \bullet, x\right) \quad\left(\text { as } x \in F^{\bullet}\right) \\
& =\left(x^{\bullet} \cup x_{1}^{\bullet} \cup \cdots \cup x_{n}^{\bullet}, x\right) \\
& =\overline{x_{1} \eta} \cdots \overline{x_{n} \eta}(x \eta)
\end{aligned}
$$

Similarly, $\left(F^{\bullet}, 1\right)=\overline{x_{1} \eta} \cdots \overline{x_{n} \eta}$. We are thus forced to define

$$
\begin{aligned}
\left(F^{\bullet}, x\right) \psi & =\overline{x_{1} f} \ldots \overline{x_{n} f}(x f) \\
\left(F^{\bullet}, 1\right) \psi & =\overline{x_{1} f} \ldots \overline{x_{n} f}
\end{aligned}
$$

which establishes uniqueness. We must show that such a definition of $\psi$ is independent of the choice of $F$ and results in a homomorphism of guarded semigroups which extends $f$.

If $y \in F^{\bullet}$ with $y \notin F=\left\{x_{1}, \ldots, x_{n}\right\} \subset S$, it follows from the definition of the closure operator that there exist $t \in S, 1 \leq i \leq n$ with $y t=x_{i} \in F$. As $\overline{(y t) f}=\overline{(y f)(t f)} \leq \overline{y f}, \overline{x_{1} f} \ldots \overline{x_{n} f} \overline{y f}=$ $\overline{x_{1} f} \ldots \overline{x_{n} f}$. Hence if $G=\left\{y_{1}, \ldots y_{m}\right\}$ with $G^{\bullet}=F^{\bullet}$ then $\overline{x_{1} f} \ldots \overline{x_{n} f} \overline{y_{1} f} \ldots \overline{y_{m} f}=\overline{x_{1} f} \ldots \overline{x_{n} f}$. Arguing symmetrically,

$$
\overline{y_{1} f} \ldots \overline{y_{m} f}=\overline{x_{1} f} \ldots \overline{x_{n} f} \overline{y_{1} f} \ldots \overline{y_{m} f}=\overline{x_{1} f} \ldots \overline{x_{n} f}
$$

so $\psi$ is well defined.

$\psi$ extends $f$ since $x \eta \psi=\left(x^{\bullet}, x\right) \psi=\overline{x f} x f=x f . \psi$ preserves guards since if $x \neq 1, \overline{\left(F^{\bullet}, x\right)} \psi=$ $\left(F^{\bullet}, 1\right) \psi=\overline{x_{1} f} \ldots \overline{x_{n} f}=\overline{x_{1} f} \ldots \overline{x_{n} f} \overline{x f}\left(\right.$ as $\left.x \in F^{\bullet}\right)=\overline{\overline{x_{1} f} \ldots \overline{x_{n} f}(x f)}(\boldsymbol{g} .3)=\overline{\left(F^{\bullet}, x\right) \psi}$. Similarly, $\left(F^{\bullet}, 1\right) \psi=\overline{x_{1} f} \ldots \overline{x_{n} f}=\overline{\overline{x_{1} f} \ldots \overline{x_{n} f}}=\overline{\left(F^{\bullet}, 1\right) \psi}$.

It remains to show $\psi$ is a semigroup homomorphism. If $x \neq 1 \neq y, F=\left\{a_{1}, \ldots, a_{m}\right\}, G=$ $\left\{b_{1}, \ldots, b_{n}\right\}$,

$$
\begin{aligned}
\left(\left(F^{\bullet}, x\right)\left(G^{\bullet}, y\right)\right) \psi & =\left((F \cup x G)^{\bullet}, x y\right) \psi \\
& =\overline{a_{1} f} \cdots \overline{a_{m} f} \frac{x b_{1} f}{\cdots} \overline{x b_{n} f}(x y) f
\end{aligned}
$$

whereas $n$ uses of $(\boldsymbol{g} .4)$ give

$$
\begin{aligned}
\left(F^{\bullet}, x\right) \psi\left(G^{\bullet}, y\right) \psi & =\overline{a_{1} f} \cdots \overline{a_{m} f}(x f) \overline{b_{1} f} \cdots \overline{b_{n} f}(y f) \\
& =\overline{a_{1} f} \cdots \overline{a_{m} f} \overline{x b_{1} f}(x f) \overline{b_{2} f} \cdots \overline{b_{n} f}(y f) \\
& \cdots \overline{a_{1} f} \cdots \overline{a_{m} f} \overline{x b_{1} f} \cdots \overline{x b_{n} f}(x f)(y f)
\end{aligned}
$$

and these results are the same because $f$ is a semigroup homomorphism. The other cases $x=1 \neq y$, $x \neq 1=y, x=1=y$ are similar.

For $X$ a set, let $X^{+}$be the free semigroup generated by $X$ and let $X^{*}=\left(X^{+}\right)^{1}$ denote the free monoid generated by $X$. 
Corollary 6.6 The free $\boldsymbol{g}$-semigroup generated by a set $X$ is the set of all $(Q, w)$ with $Q \subset X^{+}$finite and prefix-closed and $w \in X^{*}$ such that $w \in Q \cup\{1\}$ with multiplication $(Q, w)(R, v)=(Q \cup w R, w v)$, $\operatorname{guard} \overline{(Q, r)}=(Q, 1)$ and inclusion of the generators $x \mapsto(\{x\}, x)$.

\section{An Alternate Equational Description}

Recall that a right normal band is a band satisfying $x y a=y x a$.

Definition 7.1 Let $\mathcal{G S}_{\text {lu }}$ be the variety of guarded semigroups with specified left unit $u, u x=x$ for all $x$. Homomorphisms must preserve $u$.

Because $S^{1}$ has 1 as left unit for any $\boldsymbol{g}$-semigroup $S$, a characterization of this variety is very nearly a characterization of guarded semigroups. We now determine $\mathcal{G S}_{l u}$ entirely with semigroups and no explicit guard. The trick is to use two semigroup structures!

Proposition $7.2 \mathcal{G S}_{l u}$ may be presented as the variety of all $(S, \cdot, u, \star)$ where $(S, \cdot)$ is a semigroup (write $x y$ for $x \cdot y$ ), $u$ is a left unit for $(S, \cdot)$ and $(S, \star)$ is a right normal band subject to the three equations

$(\mathrm{g} \star .1) \quad u \star x=x$

$(\mathbf{g} \star .2)(y \star z) x=y \star(z x)$

$(\mathrm{g} \star .3) x(y \star u)=(x y) \star x$

Proof First let $S$ be a $\boldsymbol{g}$-semigroup and define

$$
x \star y=\bar{x} y
$$

Then $(x \star y) \star z=\bar{x} y z=\bar{x} \bar{y} z=x \star(y \star z) ; x \star x=\bar{x} x=x ; x \star y \star a=\bar{x} \bar{y} a=\bar{y} \bar{x} a=y \star x \star a$, so $(S, \star)$ is a right normal band. Observe

$$
\bar{x}=u \bar{x}=\overline{u x} u=\bar{x} u
$$

Setting $x=u, \bar{u}=\bar{u} u=u$. We check $(\boldsymbol{g} \star .1, \boldsymbol{g} \star .1, \boldsymbol{g} \star .3) . u \star x=\bar{u} y=u y=y ;(y \star z) x=\bar{y} z x=$ $y \star(z x) ; x(y \star u)=x \bar{y} u=x \bar{y}=\overline{x y} x=(x y) \star x$. Conversely, consider $(S, \cdot, u, \star)$ with $(S, \star)$ a right normal band such that $(\boldsymbol{g} \star .1, \boldsymbol{g} \star .1, \boldsymbol{g} \star .3)$ hold. Define

$$
\bar{x}=x \star u
$$

and check $(\boldsymbol{g} \cdot 1, \ldots, \boldsymbol{g} \cdot 4) . \bar{x} x=(x \star u) x=x \star(u x)=x \star x=x ; \bar{x} \bar{y}=(x \star u)(y \star u)=x \star(u(y \star u))=$ $x \star y \star u=y \star x \star u$ (right normal) $=\ldots=\bar{y} \bar{x} ; \overline{\bar{x} y}=((x \star u) y) \star u=x \star(u y) \star u=x \star y \star u=$ (as above) $\bar{x} \bar{y} ; x \bar{y}=x(y \star u)=(x y) \star x=(x y) \star(u x)=(x y \star u) x=\overline{x y} x$. To conclude the proof we must show these passages are inverse. If $(S, \cdot, u, \overline{(-)} \mapsto(S, \cdot, u, \star) \mapsto(S, \cdot, u, \widehat{(-)})$ then $\hat{x}=x \star u=\bar{x} u=\bar{x}$. If $(S, \cdot, u, \star) \mapsto(S, \cdot, u, \overline{(-)}) \mapsto(S, \cdot, u, \star \star)$ then $x \star \star y=\bar{x} y=(x \star u) y=x \star u \star y=x \star y$. 


\section{Banded Semigroups}

As we have seen in the previous section, if a $\boldsymbol{g}$-semigroup has a left unit $u$, the auxiliary operation $x \star y=\bar{x} y$ is a right normal band with $\bar{x}=x \star u$. Even without a left unit, we can consider the variety in binary $x \star y$ and unary $\bar{x}$ satisfying all equations in these operations which hold in all $\boldsymbol{g}$-semigroups. The algebras in this variety are called banded semigroups. We begin with a formal definition in terms of four equations which, it turns out, entail all the others. Later in the section we provide a characterization of those bands $(S, \star)$ which admit a the structure of a banded semigroup.

Definition 8.1 A banded semigroup, $\boldsymbol{g} \star$-band for short, is $(S, \star,-)$ where $(S, \star)$ is a semigroup and $x \mapsto \bar{x}$ is a unary operation subject to the previous equations $(\boldsymbol{g} .1, \boldsymbol{g} .2, \boldsymbol{g} .3)$ as well as

$(g \star .1) \quad x \star y=\bar{x} \star y$

The variety of all $\boldsymbol{g} \star$-bands will be denoted $\mathcal{G}_{\star} \mathcal{B}$. Homomorphisms here preserve $x \star y$ and $\bar{x}$ and we call them $\boldsymbol{g} \star$-homomorphisms. A $\boldsymbol{g} \star$ subband of a $\boldsymbol{g} \star$-band is a subsemigroup closed under $\bar{x}$ and such is $\boldsymbol{g} \star-$ full is it contains $\bar{x}$ for arbitrary $x$.

Every guarded semigroup induces a canonical banded semigroup structure on the same set as follows.

Proposition $8.2 \star: \mathcal{G S} \rightarrow \mathcal{G}_{\star} \mathcal{B}$ defined by

$$
\star(S, \cdot,-)=(S, \star,-), \quad x \star y=\bar{x} y
$$

is a well-defined functor.

Proof For $(\boldsymbol{g} .1, \boldsymbol{g} .2, \boldsymbol{g} .3), \bar{x} \star x=\overline{\bar{x}} x=\bar{x} x=x ; \bar{x} \star \bar{y}=\overline{\bar{x}} \bar{y}=\bar{x} \bar{y}=\bar{y} \bar{x}=\cdots=\bar{y} \star \bar{x}$; $\overline{\bar{x} \star y}=\overline{\bar{x} y}=\overline{\bar{x} y}=\bar{x} \bar{y}=\bar{x} \star \bar{y}$. $(\boldsymbol{g} \star .1)$ is trivial as $x \star y=\bar{x} y=\overline{\bar{x}} y=\bar{x} \star y$. If $f$ is a $\boldsymbol{g}$-homomorphism, $(x \star y) f=(\bar{x} y) f=\overline{x f}(y f)=x f \star y f$.

Proposition 8.3 A $\boldsymbol{g} \star$-band is a right normal band and the following hold:

$(g \star .2) \quad \overline{\bar{x}}=\bar{x}$

$(g \star .3) \quad \overline{x \star y}=\bar{x} \star \bar{y}$

$(g \star .4) \quad x \star \bar{y}=y \star \bar{x}$

Proof $x \star x=\bar{x} \star x=x ; x \star y \star a=\overline{\bar{x} \star y} \star a=\bar{x} \star \bar{y} \star a=\bar{y} \star \bar{x} \star a=\cdots=y \star x \star a$, so $S$ is a right normal band. $(\boldsymbol{g} \star .2)$ is the same as $(\boldsymbol{g} .7)$ which follows from $(\boldsymbol{g} \cdot 1, \boldsymbol{g} .2, \boldsymbol{g} .3)$. For $(\boldsymbol{g} \star .3)$, $\overline{x \star y}=\overline{\bar{x} \star y}=\bar{x} \star \bar{y}$; For $(\boldsymbol{g} \star .4), x \star \bar{y}=\bar{x} \star \bar{y}=\bar{y} \star \bar{x}=y \star \bar{x}$.

If $S, T$ are $\boldsymbol{g} \star$-bands, the set [S,T] of all $\boldsymbol{g} \star$-homomorphisms from $S$ to $T$ is a banded semigroup with the pointwise operations, as is routine to check. It follows from the theorem of Linton [18] that $\mathcal{G}_{\star} \mathcal{B}$ is a closed category with the appropriate tensor product and that the left and right inner translations of $S$ as well as $x \mapsto \bar{x}$ are $\boldsymbol{g} \star$-endomorphisms.

For $S$ a $\boldsymbol{g} \star$-band, denote

$$
\boldsymbol{g}_{\star}(S)=\{x: x=\bar{x}\}=\{\bar{x}: x \in S\}
$$

By the preceding proposition, $\boldsymbol{g}_{\star}(S)$ is a semilattice with infimum $x \star y$. The semilattice $\boldsymbol{g}_{\star}(\star S)$ of $\star S$ coincides with $\boldsymbol{g}(S)$. 
Proposition 8.4 Let $(S, \star,-)$ be a $\boldsymbol{g} \star$-band. Then

$$
x \leq y \Leftrightarrow x \star y=x
$$

is a compatible partial order on $S$ satisfying

$(g \star .5) \quad a \star x \leq x$

$(g \star .6) \quad \bar{x}=\bar{y}$ and $x \leq y \Leftrightarrow x=y$

With respect to this order, every semigroup homomorphism between $\boldsymbol{g} \star$-bands is monotone. Moreover, the negative cone $N$ satisfies

$$
N=Z(S, \star) \subset \boldsymbol{g}_{\star}(S)
$$

where $Z(S, \star)$ is the semigroup center.

Proof Since $x \leq y \Leftrightarrow \bar{x} y=x$, the proof that $\leq$ is a partial order and that $x \leq y \Leftrightarrow a \star x \leq a \star y$ from Proposition 3.2 works here as well since $(\boldsymbol{g} .4)$ is not used there. To see $x \star a \leq y \star a$ use right normality: $x \star a \star y \star a=x \star y \star a=x \star a . \quad(\boldsymbol{g} \star .5)$ is trivial: $a \star x \star x=a \star x$. For $(\boldsymbol{g} \star 6), x=x \star y=\bar{x} \star y=\bar{y} \star y=y$. If $f$ is a semigroup homomorphism and $x \leq y$ then $x \star y=x \Rightarrow x f=(x \star y) f=(x f) \star(y f)$ so that $x f \leq y f$. By $(\boldsymbol{g} \star .5), N=\{a: \forall x, x \star a \leq x\}$. If $a \in N$ then $a=\bar{a} \star a \leq \bar{a}$ so $a=\bar{a}$ by $(\boldsymbol{g} \star .6)$ and this shows $N \subset \boldsymbol{g}_{\star}(S)$. For $a \in N, x \in S$, as $x \star a \leq x$ we have

$$
x \star a=x \star a \star x=a \star x \star x=a \star x
$$

so $a \in Z(S, \star)$. If $a \in Z(S, \star), x \in S$, then $x \star a \star x=x \star x \star a=x \star a$ so $x \star a \leq x$ and $a \in N$.

Example 8.5 If the $\boldsymbol{g}$-semigroup $S$ is a semilattice then $\star S=S$, that is, $x \star y=\bar{x} y=x y$. Thus a semilattice satisfies all five equations $(\boldsymbol{g} .1, \ldots, \boldsymbol{g} \cdot 4, \boldsymbol{g} \star .1)$. Conversely, if $(S, \star,-)$ satisfies all five equations then it is a semilattice.

To see this, first observe

$$
x \star y \star x=\overline{x \star y} \star x=x \star \bar{y}=\bar{x} \star \bar{y}=\bar{y} \star \bar{x}=\cdots=y \star x \star y
$$

But then,

$$
x \star y=x \star y \star y=y \star x \star y=x \star y \star x=y \star x \star x=y \star x
$$

and $S$ is a commutative band. Moreover,

$$
x=\bar{x} \star x=x \star \bar{x}=\bar{x} \star \bar{x}=\bar{x}
$$

so $S$ is a semilattice.

Example 8.6 Let $S$ a any monoid qua $\boldsymbol{g}$-semigroup with $\bar{x}=1$. Then $\star S$ is a right zero semigroup since $x \star y=\bar{x} y=1 y=y$

Indeed, for any set $S$ with $x_{0} \in S, x \star y=y, \bar{x}=x_{0}$ gives a $\boldsymbol{g} \star$-band. If a $\boldsymbol{g} \star$-band is right zero then $\bar{x}$ must be constant since

$$
\bar{x}=\bar{y} \star \bar{x}=\bar{x} \star \bar{y}=\bar{y}
$$

Semilattices and right zero $\boldsymbol{g} \star$-bands generate the variety of all $\boldsymbol{g} \star$-bands. This is an immediate corollary of the construction of the free $\boldsymbol{g} \star$-band in the proof of the next proposition. 
Proposition 8.7 Let $X$ be a set and set $X_{\perp}=X \cup\{\perp\}$ where $\perp \notin X$. Regard $X_{\perp}$ as the right zero $\boldsymbol{g} \star$-band with $\bar{x}=\perp$. Let $P_{0}(X)$ be the semilattice of finite subsets of $X$ under union. Then

$$
J(X)=\left\{(A, x) \in P_{0}(X) \times X_{\perp}: x \in A \cup\{\perp\}\right\}
$$

is a $\boldsymbol{g} \star$-subband of the product band. Further, with respect to the inclusion

$$
\eta: X \rightarrow J(X), \quad x \mapsto(\{x\}, x)
$$

$J(X)$ is the free $\boldsymbol{g} \star$-band generated by $X$.

Proof If $(A, x),(B, y) \in J(X)$ then

$$
(A, x)(B, y)=(A \cup B, y)
$$

As $y \in B \cup\{\perp\}, y \in A \cup B \cup\{\perp\}$. Also, $\overline{(A, x)}=(\bar{A}, \bar{x})=(A, \perp)$ with $\perp \in A \cup\{\perp\}$. Thus, $J(X)$ is a full $\boldsymbol{g} \star$-subband of $P_{0}(X) \times X_{\perp}$. Now, let $S$ be an arbitrary $\boldsymbol{g} \star$-band and let $f: X \rightarrow S$ be any function. We must show that there exists unique $\boldsymbol{g} \star$-homomorphism $\psi: J(X) \rightarrow S$ with $\eta \psi=f$. Consider $(A, x) \in J(X)$. If $x \in A$ write $A=\left\{a_{1}, \ldots, a_{n-1}, x\right\}$ and observe that

$$
(A, x)=\overline{\left(\left\{a_{1}\right\}, \perp\right)} \cdots \overline{\left(\left\{a_{n-1}\right\}, \perp\right)}(\{x\}, x)=\overline{a_{1} \eta} \cdots \overline{a_{n-1} \eta}(x \eta)
$$

which forces

$$
(A, x) \psi=\overline{a_{1} f} \ldots \overline{a_{n-1} f}(x f)
$$

Similarly, if $x=\perp$, necessarily

$$
(A, \perp) \psi=\overline{a_{1} f} \ldots \overline{a_{n} f}
$$

where $A=\left\{a_{1}, \ldots, a_{n}\right\}$. It is routine to check that such $\psi$ is a $\boldsymbol{g} \star$-homomorphism.

We are now able to prove the promised theorem connecting the equational theory of $\boldsymbol{g} \star$-bands to guarded semigroups.

Theorem 8.8 Let $\mathcal{E}$ be the set of all equations in the operations $x \star y$ and $\bar{x}$ which hold for $\star S$ as $S$ ranges over all guarded semigroups. Let $\mathcal{F}$ be the set of all equations in $x \star y$ and $\bar{x}$ which hold for all $\boldsymbol{g} \star$-bands. Then $\mathcal{E}=\mathcal{F}$.

Proof $\mathcal{F} \subset \mathcal{E}$ by Proposition 8.2. For the converse it suffices to show $J(X)$ has form $\star S$ since then all equations in $\mathcal{E}$ hold for all free $\boldsymbol{g} \star$-bands and hence for all $\boldsymbol{g} \star$-bands. Let $X$ have any semigroup structure, left or right zero for example. Form the monoid $X^{1}$ and let $X_{\perp}=X^{1}$ with $\perp=1$. As is true for any monoid, $X^{1}$ acts on $P_{0}(x)$ (the finite subsets of $X$, recall) by $x \cdot A=x A$, where $x A=\{x a: a \in A\}$, as is routine to check. This gives rise to the semidirect product $\boldsymbol{g}$-semigroup $P_{0}(X) \bowtie X^{1}$ with operations

$$
\begin{aligned}
(A, x)(B, y) & =(A \cup x B, x y) \\
\overline{(A, x)} & =(A, 1)
\end{aligned}
$$

But observe that $\star\left(P_{0}(X) \bowtie X^{1}\right)$ has multiplication

$$
(A, x) \star(B, y)=(A, 1)(B, y)=(A \cup B, y)
$$

which is the product $\boldsymbol{g} \star$-band structure as in Proposition 8.7. Moreover, $J(X)$ is a $\boldsymbol{g}$-subsemigroup of $P_{0}(X) \bowtie X^{1}$ because if $y \in B$ then $x y \in x B$.

The semigroups that underlie $\boldsymbol{g}$-semigroups do not have very special structure since all monoids are in this class. It is more interesting to ask which right normal bands can be a $\boldsymbol{g} \star$-band for 
some $\bar{x}$. The theorem of Yamada and Kimura [26] gives that a band is a strong semilattice of rectangular bands if and only if axya=ayxa. It is known (See [16, Corollary 5.18]) that the right normal bands are precisely the strong semilattices of right zero semigroups. See [1, Pages $402 \mathrm{ff}]$ for information about semilattices of right zero semigroups. The next theorem gives necessary and sufficient conditions in this style for the semigroups at hand.

Definition 8.9 Let the semigroup $S$ be a semilattice of semigroups via a semilattice-valued surjective homomorphism $\psi: S \rightarrow L$. Say that such $S$ is a split semilattice of semigroups if there exists a homomorphism $i: L \rightarrow S$ with $i \psi=i d_{L}$.

Up to isomorphism, we may replace $L$ by its image under $i$ so that $L$ is a subsemigroup of $\mathrm{S}$ and $\psi$ is a surjective homomorphism with $x \psi=x$ for $x \in L$.

Theorem 8.10 For a semigroup $(S, \star)$, equivalent are

(1) There exists a unary operation $\bar{x}$ on $S$ making $(S, \star,-)$ a $\boldsymbol{g} \star$-band.

(2) $S$ is a split strong semilattice of right zero semigroups.

Proof $\quad(1) \Rightarrow(2)$ : Let $L=\boldsymbol{g}_{\star}(S)$, a semilattice with multiplication as infimum whose partial order is the restriction of the partial order of Proposition 8.4. Define $\psi: S \rightarrow L$ by $x \psi=\bar{x}$. By $(\boldsymbol{g} \star .2$, $\boldsymbol{g} \star .3), \psi$ is a semigroup homomorphism and $x \in L \Rightarrow x \psi=x$. For $e \in L$ let $S_{e}=\{x: \bar{x}=e\}$. For $x, y \in S_{e}, x \star y=\bar{x} \star y=\bar{y} \star y=y$, so $S_{e}$ is a right zero semigroup. So far, $S$ is a split semilattice of right zero semigroups. For $e \geq f$ define $\psi_{e f}: S_{e} \rightarrow S_{f}$ by $x \psi_{e f}=f \star x$. As $\overline{f \star x}=\bar{f} \star \bar{x}=f \star e=f$, this is well-defined. If $e \geq f \geq g$ then $\psi_{e f} \psi_{f g}=\psi_{e g}$ because $x \psi_{e f} \psi_{f g}=(f \star x) \psi_{f g}=g \star f \star x=g \star x=x \psi_{e g}$. Each $\psi_{e} f$ is a semigroup homomorphism because any function between right zero semigroups is. For $x \in S_{e}, y \in S_{f}$, we evaluate the the $S_{e \star f}$ product

$$
\left(x \psi_{e, e \star f}\right) \star\left(y \psi_{f, e \star f}\right)=y \psi_{f, e \star f} \text { (right zero) }=e \star f \star y=e \star \bar{y} \star y=e \star y=\bar{x} \star y=x \star y
$$

This shows that $S$ is a split strong semilattice of right zero semigroups.

$(2) \Rightarrow(1)$ : Define $\bar{x}=x \psi$. For $x \in S_{e}, y \in S_{f}$,

$$
x \star y=\left(x \psi_{e, e \star f}\right) \star\left(y \psi_{f, e \star f}\right)=\left(\bar{x} \psi_{e, e \star f}\right) \star\left(y \psi_{f, e \star f}\right) \quad(\text { right zero })=\bar{x} \star y
$$

so $(\boldsymbol{g} \star .1)$ holds. As $x, \bar{x} \in S_{\bar{x}}, \bar{x} \star x=x$ by right zero. By semilattice, $\bar{x} \star \bar{y}=\bar{y} \star \bar{x}$. Finally, as $\psi$ is a semigroup homomorphism, $\overline{\bar{x} \star y}=\overline{x \star y}=\bar{x} \star \bar{y}$.

Proposition 8.11 Let $\mathcal{D}$ be the class of all semigroups $(S, \star)$ characterized in the previous theorem, that is, for which there exists $x \mapsto \bar{x}$ making $S$ a $\boldsymbol{g} \star$-band. Then $\mathcal{D}$ is not a variety, but the variety it generates is that of all right normal bands.

Proof The lattice of varieties of bands is well-understood ([5]). The immediate predecessors of the variety of right normal bands are the right zero semigroups and semilattices. Since clearly $\mathcal{D}$ is contained in neither of these (consider $\star S$ for, say, $S=P f n(X)$ ), $\mathcal{D}$ generates all right normal bands. An example of a right normal band not in $\mathcal{D}$ is the free one on two generators, $S=\{a, b, a \star b, b \star a\}$. For if $S$ were a $\boldsymbol{g} \star$-band with respect to $\bar{x}$, for $x=a, b, a \star b, b \star a, x \star a$ is, respectively, $a, b \star a, b \star a, b \star a$ so the only solution of $x \star a=a$ is $x=a$ and $\bar{a}=a$. Similarly, $\bar{b}=b$. But as $a \star b \neq b \star a$ whereas $\bar{a} \star \bar{b}=\bar{b} \star \bar{a}$, we have a contradiction. 


\section{Banded Semigroups with Given Semilattice}

Let $L$ be a fixed meet semilattice. In this section we show how to construct the general banded semigroup $S$ with $\boldsymbol{g}_{\star}(S) \cong L$. We begin with some generalities.

Consider $(\mathcal{C}, 1)$ where $\mathcal{C}$ is a category and 1 is a chosen terminal object of $\mathcal{C}$. For $X$ an object of $\mathcal{C}$, we write $x \in X$ to abbreviate that $x$ is a morphism $x: 1 \rightarrow X$. Such $x$ is called a global element of $X$. The category $(\mathcal{C}, 1)_{\star}$ has as objects all pairs $(X, x)$ with $x \in X$ and has as morphisms $f:(X, x) \rightarrow(Y, y)$ all morphisms $f: X \rightarrow Y$ in $\mathcal{C}$ such that $x f=y$.

$L^{o p}$ is the category whose objects are the elements of $L$ and for which there is at most one morphism $x \rightarrow y$ with such existing precisely when $x \geq y$. Reflexivity gives identity morphisms and transitivity gives the composition. For $\mathcal{S}$ the category of sets and total transformations, let $\mathcal{S}^{L^{o p}}$ be the usual functor category with functors $L^{o p} \rightarrow \mathcal{S}$ as objects and natural transformations as morphisms.

Let 1 be a chosen singleton set in $\mathcal{S}$ and then choose the functor constantly 1 as a terminal object of $\mathcal{S}^{L^{o p}}$, also denoted 1 . We now show that objects in $\left(\mathcal{S}^{L^{o p}}, 1\right)_{\star}$ construct banded semigroups. For $F \in \mathcal{S}^{L^{o p}}$ and $e \geq f \in L$ denote the corresponding function as $F_{e, f}: e F \rightarrow f F$. A a global element $\tau \in F$ amounts to a family $\tau_{e} \in e F$ such that whenever $e \geq f, \tau_{e} F_{e, f}=\tau_{f}$.

Theorem 9.1 Let $(F, \tau) \in\left(\mathcal{S}^{L^{o p}}, 1\right)_{\star}$. Let $S$ be the disjoint union

$$
S=\coprod(e F: e \in L)
$$

and for $x \in e F$ define $\bar{x}=\tau_{e} \in e F \subset S$. For $x \in e F, y \in f F$, define

$$
x \star y=y F_{f, e f} \in(e f) F \subset S
$$

Then $S=(S, \star,-)$ is a $\boldsymbol{g} \star$-band with $\boldsymbol{g}_{\star}(S) \cong L$ and every such $\boldsymbol{g} \star$-band arises this way.

Proof Let $x \in e F, y \in f F, z \in g F$. To show associativity,

$$
\begin{aligned}
(x \star y) \star z & =\left(y F_{f, e f}\right) \star z=z F_{g, e f g} \\
& =z F_{g, f g} F_{f g, e f g} \text { (functor) } \\
& =x \star z F_{g, f g}=x \star(y \star z)
\end{aligned}
$$

For $(\boldsymbol{g} .1)$,

$$
\begin{aligned}
\bar{x} \star x & =\tau_{e} \star x=x F_{e, e} \\
& =x i d_{e F} \quad \text { (functor) } \\
& =x
\end{aligned}
$$

For (g.2),

$$
\begin{aligned}
\bar{x} \star \bar{y} & =\tau_{e} \star \tau_{f}=\tau_{f} F_{f, e f} \\
& =\tau_{e f}(\tau \text { is natural }) \\
& =\tau_{f e}=\bar{y} \star \bar{x}
\end{aligned}
$$

For (g.3),

$$
\begin{aligned}
\overline{\bar{x} \star y} & =\overline{x \star y} \\
& =\tau_{e f} \quad(\text { as } x \star y \in(e f) F) \\
& =\bar{x} \star \bar{y} \quad(\text { by }(\boldsymbol{g} .2) \text { proof })
\end{aligned}
$$


For $(\boldsymbol{g} \star .1)$,

$$
\begin{aligned}
x \star y & =y F_{f, e f} \\
& =\tau_{e} \star y \quad\left(\text { as } \tau_{e} \in e F\right) \\
& =\bar{x} \star y
\end{aligned}
$$

By construction, $L \rightarrow \boldsymbol{g}_{\star}(S), e \mapsto \tau_{e}$ is bijective. The proof of $(\boldsymbol{g} .2)$ showed $\tau_{e} \star \tau_{f}=\tau_{e \star f}$, so this map is an order isomorphism. Now let $S$ be a $\boldsymbol{g} \star$-band with $\boldsymbol{g} \star(S)=L$. Define $e F=\{x: \bar{x}=e\}$ and, for $e \geq f, F_{e, f}: e F \rightarrow f F, x \mapsto f \star x$. As $\overline{f \star x}=\bar{f} \star \bar{x}=f \star e=f$, this is well defined. If $e \geq f \geq g, x F_{e \star f} F_{f \star g}=(f \star x) F_{f \star g}=g \star(f \star x)=(g \star f) \star x=g \star x$ (as $\left.f \geq g\right)=x F_{e, g}$ so $F$ preserves composition. $x F_{e, e}=e \star x=\bar{x} \star x=x$ so $F$ is a functor. $\tau_{e}=e$ is natural as $\tau_{e} F_{e, g}=f \star e=f=\tau_{f}$.

We leave it as an exercise for the reader to show that the above construction establishes an equivalence of categories between $\left(\mathcal{S}^{L^{o p}}, 1\right)_{\star}$ and the category of banded semigroups $S$ with $g \star(S)=$ $L$, where the morphisms of the latter are $\boldsymbol{g} \star$-homomorphisms which restrict to the identity on $L$.

\section{Open Questions}

1. Characterize $\boldsymbol{g}$-proper guarded semigroups.

2. Determine for which guarded semigroups, the $\boldsymbol{g}$-semigroup natural order coincides with the Mitsch partial order.

3. Forgetful functors between quasivarieties must have left adjoints. What are explicit constructions for the left adjoints, for example, of the functors $\star: \mathcal{G S} \longrightarrow \mathcal{G} \star \mathcal{B}, \boldsymbol{g}$-proper $\longrightarrow \mathcal{G S}$, $\mathcal{G S}_{\text {lu }} \longrightarrow \mathcal{G S}$, Inverse $\longrightarrow \mathcal{G S}$ ? What are the surjective reflections for Left Ample $\longrightarrow \mathcal{G S}$, Weakly Left Ample $\longrightarrow \mathcal{G S}$ ?

4. Characterize guarded semigroups as a class of partially ordered semigroups.

5. Is there a banded semigroup which is not the underlying banded semigroup of a guarded semigroup?

\section{References}

[1] M. Ćirić and S. Bogdanović, Theory of greatest decompositions of semigroups (a survey), Filomat (Niš) 9:3, 1995, 385-426.

[2] J. R. B. Cockett, and S. Lack, Restriction categories I: categories of partial maps, Theoretical Computer Science 270, 2002, 223-259.

[3] M. R. Darnel, Theory of Lattice-Ordered Groups, Marcel Dekker, Inc., 1995.

[4] R. A. Di Paola and A. Heller, Dominical categories: recursion theory without elements, Journal of Symbolic Logic 52, 1987, 595-635.

[5] C. F. Fennemore, All varieties of bands I, II, Mathematische Nachricte 48, 1971, 237-252, $253-262$.

[6] J. Fountain, Right PP monoids with central idempotents, Semigroup Forum 13, 1977, 229-237.

[7] J. Fountain, A class of right PP monoids, Quarterly Journal of Mathematics 28, 1977, 285-300. 
[8] J. Fountain, Adequate semigroups, Proceedings of the Edinburgh Mathematical Society 22, 1979, 113-125.

[9] J. Fountain and G. M. S. Gomes, Left proper E-dense monoids, Journal of Pure and Applied Algebra 80, 1992, 1-27.

[10] J. Fountain and G. M. S. Gomes, Proper left type-A monoids revisited, Glasgow Math. Journal 1993, 293-306.

[11] J. Fountain and G. M. S. Gomes, Proper left type-A covers, Portugaliae Mathematica 51, 1994, $305-319$

[12] G. M. S. Gomes and V. Gould, Proper weakly left ample semigroups, International Journal of Algebra and Computation 9, 1999, 721-739.

[13] G. M. S. Gomes and V. Gould, Graph expansions of unipotent monoids, Communications in Algebra 28, 2000, 447-463.

[14] G. M. S. Gomes and V. Gould, Finite proper covers in a class of finite semigroups with commuting idempotents, Semigroup Forum 66, 2003, 433-454.

[15] V. Gould, Right cancellative and left ample monoids: quasivarieties and proper covers, Journal of Algebra 228, 2000, 428-456.

[16] J. M. Howie, An Introduction to Semigroup Theory, Academic Press, 1976.

[17] M. Kilp, Commutative monoids all of whose principal ideals are projective, Semigroup Forum 6, 1973, 334-339.

[18] F. E. J. Linton, Autonomous equational categories, Journal of Mathematics and Mechanics $15,1968,637-642$.

[19] F. Lorrain, Notes on topological spaces with minimum neighborhoods, American Mathematical Monthly 76, 616-627.

[20] S. W. Margolis and J. E. Pin, Inverse semigroups and extensions of groups by semilattices, Journal of Algebra 110, 1987, 277-297.

[21] D. B. McAlister, Groups, semilattices and inverse semigroups, Transactions of the American Mathematical Society 192, 1974, 227-244.

[22] D. B. McAlister, Groups, semilattices and inverse semigroups. II, Transactions of the American Mathematical Society 192, 1974, 351-370.

[23] D. B. McAlister, Amenably ordered inverse semigroups, Journal of Algebra 65, 1980, 118-146.

[24] H. Mitsch, A natural partial order for semigroups, Proceedings of the American Mathematical Society 97, 1986, 384-388.

[25] H. E. Scheiblich, Free inverse semigroups, Proceedings of the American Mathematical Society $38,1973,1-7$.

[26] M. Yamada and N. Kimura, Note on idempotent semigroups II, Proc. Japan Acad. 34, 1958, $110-112$. 
Department of Mathematics and Statistics

University of Massachusetts

Amherst, MA 01003

USA 